\title{
PHASE TRANSITION AT THE METRIC ELASTIC UNIVERSE
}

\author{
ALEXANDER GUSEV \\ Department of General Relativity and Astronomy, \\ Kazan State University \\ 18, Lenin str., Kazan, 420008, Russia
}

At the last time the concept of the curved space-time as the some medium with stress tensor $\sigma_{\alpha \beta}$ on the right part of Einstein equation is extensively studied in the frame of the Sakharov - Wheeler metric elasticity(Sakharov (1967), Wheeler (1970)). The physical cosmology pre- dicts a different phase transitions (Linde (1990), Guth (1991)). In the frame of Relativistic Theory of Finite Deformations (RTFD) (Gusev (1986)) the transition from the initial state $g_{\alpha \beta}^{o}$ of the Universe (Minkowskian's vacuum, quasi-vacuum(Gliner (1965), Zel'dovich (1968)) to the final state $g_{\alpha \beta}$ of the Universe(Friedmann space, de Sitter space) has the form of phase transition(Gusev (1989) which is connected with different space-time symmetry of the initial and final states of Universe(from the point of view of isometric group $G_{n}$ of space). In the RTFD (Gusev (1983), Gusev (1989)) the space-time is described by deformation tensor $\epsilon_{\alpha \beta}=g_{\alpha \beta}-g_{\alpha \beta}^{\circ}$ of the three-dimensional surfaces, and the Einstein's equations are viewed as the constitutive relations between the deformations $\epsilon_{\alpha \beta}$ and stresses $\sigma_{\alpha \beta}$. The vacuum state of Universe have the visible zero physical characteristics and one is unsteady relatively quantum and topological deformations (Gunzig \& Nardone (1989), Guth (1991)). Deformations of vacuum state, identifying with empty Mikowskian's space are described the deformations tensor $\epsilon_{\alpha \beta}$, where $g_{\alpha \beta}=g_{\alpha \beta}-U_{\alpha} U_{\beta}$ the metrical tensor of deformation state of 3 -geometry on the hypersurface, which is ortogonaled to the four-velocity $U^{\alpha}, \dddot{g}_{\alpha \beta}^{o}=\delta_{\alpha}^{\gamma} \delta_{\beta}^{\zeta} g_{\gamma \zeta}$ is the 3 -geometry of initial state, $\delta_{\alpha}^{\gamma}=\delta_{\alpha}^{\gamma}-U_{\alpha} U^{\gamma}$ is a projection tensor.

The phenomenological description of thermodynamics systems" creating matter - elastic deformations of gravitational field " can be make on the basis of general theory phase transitions of 2nd kind Landau(Landau \& Lifshich (1976)). The characteristic of the phase transitions theory of 2nd kind is to vanish a some element symmetry of thermodynamics system which are 
connected with appearance a order parameter $\eta$ and to creating at the nonsymmetrical phase the some new macroscopical physical values, which are vanished at the symmetrical phase. For a description of macroscopical properties media (Prigogin (1988)) a depende- nce of free energy $\mathrm{F}=1 / 2 \sigma^{\alpha \beta} \epsilon_{\alpha \beta}$ $+\varrho$, entropy $\mathrm{S}$ of the Universe on the temperature $\mathrm{T}$, deformation tensor $\epsilon_{\alpha \beta}$, order parameter $\eta=\sqrt{T-T_{c}}=\sqrt{1-l_{o}^{2} / R^{2}}$ is being found out. In the initial symmetrical phase (from the point of view of isometry group $G_{10}$ - Poincare's group) for $t<t_{o} R=l_{o}, \varrho=P=F=T=S=0$. In the final nonsymmetrical phase (from the point of view of isometry group $G_{7}$ of space) for $t>t_{o}$ evolution of the Universe is developing by the following law: $\mathrm{R} \rightarrow \cosh \hat{t}, \rho \rightarrow \tanh ^{2} \hat{t}$,

$$
\begin{gathered}
\mathrm{P}=\sigma_{\alpha}^{\alpha} / 3 \propto \sum_{n=1}^{6} \mathrm{C}_{n} / \mathrm{R}^{n} \\
\mathrm{~S}=-\frac{\partial \mathrm{F}}{\partial \mathrm{T}}=\mathrm{S}_{0}-a_{1} \mathrm{~T}+a_{2} \mathrm{~T}^{2}-a_{3} \mathrm{~T}^{3} \\
F=-2 a_{1}-\frac{1}{2} a_{2} \eta^{2}+\frac{3}{4} a_{1} \eta^{4}+2 a_{1}\left(1-\eta^{2}\right)^{3 / 2}-\frac{3}{2} a_{3}\left(1-\eta^{2}\right) \ln \left(1-\eta^{2}\right)
\end{gathered}
$$

where $\hat{t}=\sqrt{8 \pi a_{i s} / 3}\left(t-t_{o}\right), a_{a d}=\alpha\left(T-T_{c}\right), S_{o}$ is an initial entropy of Universe, can be equal a zero, $\alpha$ is a thermal expansion coefficient, $T_{c} \geq 0$ is a critical temperature, $a_{1}, a_{2}, a_{3}$ are a parameters of RTFD. This solution has the asymptotic of de Sitter Universe (Hoyle, Barbidge and Narlikar (1993)) The analyses of the phase portraits of one-, two- , three-parameter DS and of the bifurcation space of parameters (Gusev (1989)) confirms the character of chaotic inflation(Linde (1990)) at the early times.

\section{References}

Gliner, E.B., (1965), Zhurnal eksper. i teoretich. fiziki., 49, p.542

Gunzig, E., Nardone, P., (1989), Int. J. Theor. Phys., 28, p.943

Gusev, A.V., (1983), Proc. Kazan Astr. Observatory, 47, p.126

Gusev, A.V., (1986), Proc. Kazan Astr. Observatory, 50, p.33

Gusev, A.V., (1989), Proc. Kazan Astr. Observatory, 52, p.114

Guth, A.H., (1991), Physica Scripta, 36, p.237

Hoyle, F., Barbidge, G., Narlikar, J.V. (1993), Ap. J, 410, p.437

Landau, L.D., Lifshitz, E.M., (1976), Statisticheskay fizika, Moscow, Nauka

Linde, A.D., (1990), Elementary Particle Physics and Inflationary Cosmology, Moscow, Nauka.

Misner C.W., Thorne K.S. and Wheeler J. A., (1973), Gravitation, San Francisco, Freeman

Prigogine, I. et al, (1988), Proc. Nat. Acad. Sci. USA, 85, p.7428

Sakharov, A.D., (1967), Dokl. Akad. Nauk USSR, 177, p.70

Zel'dovich, Ya.B., (1968), Usp. Phys. Nauk, 95, p.209 